\title{
The stability of spectroscopic instruments: a unified Allan variance computation scheme
}

\author{
V. Ossenkopf \\ 1 I. Physikalisches Institut der Universität zu Köln, Zülpicher Straße 77, 50937 Köln, Germany \\ e-mail: ossk@ph1.uni-koeln.de \\ 2 SRON Netherlands Institute for Space Research, PO Box 800, 9700 AV Groningen, The Netherlands \\ ${ }^{3}$ Kapteyn Astronomical Institute, University of Groningen, PO box 800, 9700 AV Groningen, The Netherlands
}

Received 4 December 2007 / Accepted 26 December 2007

\section{ABSTRACT}

\begin{abstract}
Context. The Allan variance is a standard technique to characterise the stability of spectroscopic instruments used in astronomical observations. The period for switching between source and reference measurement is often derived from the Allan minimum time. However, various methods are applied to compute the Allan variance spectrum and to use its characteristics in the setup of astronomical observations.

Aims. We propose a new approach for the computation of the Allan variance of spectrometer data combining the advantages of the two existing methods into a unified scheme. Using the Allan variance spectrum we derive the optimum strategy for symmetric observing schemes minimising the total uncertainty of the data resulting from radiometric and drift noise.

Methods. The unified Allan variance computation scheme is designed to trace total-power and spectroscopic fluctuations within the same framework. The method includes an explicit error estimate both for the individual Allan variance spectra and for the derived stability time. A new definition of the instrument stability time allows to characterise the instrument even in the case of a fluctuation spectrum shallower than $1 / f$, as measured for the total power fluctuations in high-electron-mobility transistors.

Results. A first analysis of test measurements for the HIFI instrument shows that gain fluctuations represent the main cause of instrumental instabilities leading to large differences between the stability times relevant for measurements aiming at an accurate determination of the continuum level and for purely spectroscopic measurements. Fast switching loops are needed for a reliable determination of the continuum level, while most spectroscopic measurements can be set up in such a way that baseline residuals due to spectroscopic drifts are at a lower level than the radiometric noise. We find a non-linear impact of the binning of spectrometer channels on the resulting noise and the Allan time deviating from the description in existing theoretical treatments.
\end{abstract}

Key words. methods: data analysis - methods: statistical - instrumentation: spectrographs

\section{Introduction}

All radio-astronomical measurements are affected by instabilities of the gain, the transmission function, and the internal system noise changing the absolute scale of the measured signal. To compensate for these drifts, one switches between the astronomical source and a reference signal - a known internal calibrator or a point on the blank sky - on a timescale short compared to the instabilities. Only the difference signal is used then (see e.g. Kraus 1980; Rohlfs \& Wilson 1986).

Because of the overheads introduced by switching, the optimum strategy is not to switch as fast as possible, but only as fast as necessary to suppress the drift noise. Therefore the characteristic timescales of the instabilities have to be measured. This can be done in terms of the Allan variance (Allan 1966), a powerful technique to determine the stability of general radioastronomical equipment, in particular for systems consisting of heterodyne receivers and spectrometer backends (e.g. Kooi et al. 2000). The Allan variance spectrum can be computed from any sufficiently long time series of spectrometer dumps taken at fixed instrumental settings, provided that the integration times for the individual dumps are small compared to all instabilities.

When designing the observing modes for HIFI, the heterodyne instrument of the Herschel Space Observatory, to be launched in 2008 (de Graauw \& Helmich 2000), we had to develop a reliable Allan variance computation method for characterising the instrument stability so that the precious observing time is efficiently used in astronomical observations. The technique developed here can be applied in the same way to any ground-based telescope when the instability of the atmosphere is included in the measurement by looking at blank sky through the atmosphere.

An optimum algorithm for the computation of the Allan variance spectrum should fulfil four requirements:

- detect and characterise all instabilities and their spectral variation across the measured frequency range;

- efficiently use the measured data to extract a maximum of information from a limited time series;

- provide a measure for the uncertainty of the analysis itself, i.e. include an error estimate;

- be sufficiently rapid to allow the use of the Allan variance analysis as part of a quick-look analysis of measured data.

At present, two different algorithms are widely used to compute the Allan variance which fulfil the requirements given above only partially. The spectroscopic Allan variance, as proposed by Schieder et al. (1985; see also Kooi et al. 2000), uses only one or two arbitrarily selected channels to measure total-power or spectroscopic fluctuations, thus neglecting a large amount of the measured information. The baseline Allan variance, as proposed 
by Siebertz (1998; see also Schieder et al. 2000), considers all variations across the spectrometer, but it ignores total-power drifts and allows no identification of problematic channel ranges within the spectrum.

Here, we propose a new scheme which unifies the different approaches into a single mathematical description and largely fulfils the four requirements. The request for a complete characterisation of the spectral behaviour of the possible instabilities will be translated into the need for a computation of the Allan variance independently for each backend channel, so that channel by channel variations can be detected, the influence of standing wave instabilities becomes visible, and regions of instabilities across the IF band can be identified.

The requirement for an efficient data use is fulfilled by actually using the data from all spectrometer channels and by taking into account all possible statistically independent samplings of the temporal behaviour in the analysis. This efficient use of data helps to shorten the actual time needed to acquire the data. Although a complete characterisation of the instrumental stability requires very long time series of measurements, the derivation of the Allan variance minimum and the drift index of fluctuations at time scales in the order of the Allan minimum time can be obtained from a measurement that lasts only about three Allan minimum times. Focusing on these two quantities being the actually limiting factors for the planning and the calibration of astronomical observations allows to draw significant conclusions also from reasonably short time series, thus saving observing time.

Even if the time series of measurements is too short to guarantee a complete statistical invariance of the data, we can derive an explicit error estimate for the Allan variance from the counting statistics of the data taking. With the new definition of the Allan time proposed here this also provides a direct measure for the error of the Allan stability time. The request for a fast implementation is fulfilled by the proposed convolution schemes for the measured data, either in the time domain or in the Fourier domain.

In Sect. 2 we discuss the influence of different data normalisation schemes. Section 3 summarises our algorithm for the actual Allan variance computation including the error estimate. Section 4 discusses the best definition of a stability time and the effect of the binning of spectrometer data and Sect. 5 uses the results to derive the optimum observing strategy for all symmetric astronomical observing schemes. The implications for asymmetric schemes like on-the-fly mappings will be discussed in a separate paper. Section 6 summarises our results.

\section{Data handling}

\subsection{Normalisation across spectrometers}

To perform an instrumental stability analysis a time series of spectral dumps has to be taken consisting of spectrometer count rates $c_{i}\left(t_{k}\right)$, where the index $i$ denotes the channel number across the backend and $t_{k}$ gives the time for the spectral dump with index $k$. The existing Allan variance analysis tools always assume that the readout dead time for an individual data dump is negligible relative to the integration time between two dumps. Depending on the instrumental operation, this assumption may be violated in some cases and we will discuss the effect of dead times below. We have to assume, however, that every measurement $c_{i}\left(t_{k}\right)$ covers the same integration time.

The spectrum of counts per channel $c_{i}$ on any source is always dominated by the system bandpass of the instrument which is rarely flat and may be strongly varying across the covered frequency range. Astronomical data are thus calibrated with respect to this bandpass using two reference measurements, obtained from the blank sky and one or two thermal calibration sources (Kutner \& Ulich 1981), to deduce the actual input signal. To make the stability analysis directly applicable to astronomical measurements, the same normalisation should be used instead of working with raw backend count rates. However, a full astronomical calibration is neither practical nor necessary. The bandpass can be approximated by the average signal level obtained in the measurement corrected by the zero level of the instrument. A useful normalisation of the spectra is thus provided by

$s_{i}\left(t_{k}\right)=\frac{c_{i}\left(t_{k}\right)-z_{i}}{\left\langle c_{i}\left(t_{k}\right)-z_{i}\right\rangle_{k}}$

where $z_{i}$ is the zero level of channel $i$ and the temporal average of each channel is used to normalise the signal level of that channel. This normalisation is also used in the baseline Allan variance analysis by Siebertz (1998). It results in an approximate equivalence of all backend channels so that differences in their mutual behaviour appear on the same scale. All variations are seen relative to the signal level so that they can be compared directly to calibration errors and the radiometric noise level.

\subsection{Spectroscopic versus total power normalisation}

Most fluctuations in the amplifiers or other components of the signal path lead to variations which are constant across the whole bandpass. Observations which do not switch between source and reference on a time scale short compared to the corresponding fluctuations will exhibit baseline offsets in the calibrated data. However, most astronomical heterodyne observations are not intended to obtain an accurate continuum level but for the measurement of lines on top of a constant baseline which is typically taken to be zero. In this case, only fluctuations which do not affect all channels in the same way result in a degradation of the calibrated astronomical data. Spectroscopic fluctuations change the mutual response between different channels, often seen as ripples or steps in the baseline.

Astronomical observations thus call for two different kinds of instrument stabilities, a total power stability and a spectroscopic stability. Two different types of Allan variance analyses are required. The total power Allan variance has to trace all instrumental variations. It is computed directly from the normalised spectra as given in Eq. (1). The spectroscopic Allan variance measures only instabilities deviating from a common gain variation across the whole band. In the original definition of the spectroscopic Allan variance by Schieder et al. (1985) this is accomplished by considering the difference signal between two channels $i$ and $j$. This approach, however, suffers from the arbitrariness of the selection of these channels and the impossibility to distinguish the contributions from either of the channels. To avoid these problems, we propose to use the average over the whole backend to subtract the continuum level fluctuations. We extend Eq. (1) by this difference for the spectroscopic Allan variance computation:

$$
s_{i}\left(t_{k}\right)=\frac{c_{i}\left(t_{k}\right)-z_{i}}{\left\langle c_{i}\left(t_{k}\right)-z_{i}\right\rangle_{k}}-\left\langle\frac{c_{i}\left(t_{k}\right)-z_{i}}{\left\langle c_{i}\left(t_{k}\right)-z_{i}\right\rangle_{k}}\right\rangle_{i} .
$$

This spectroscopic normalisation corresponds to the subtraction of a zero-order baseline in the Allan variance method proposed by Siebertz (1998). For long time series, the resulting spectroscopic Allan variance spectra are also equivalent to the average 

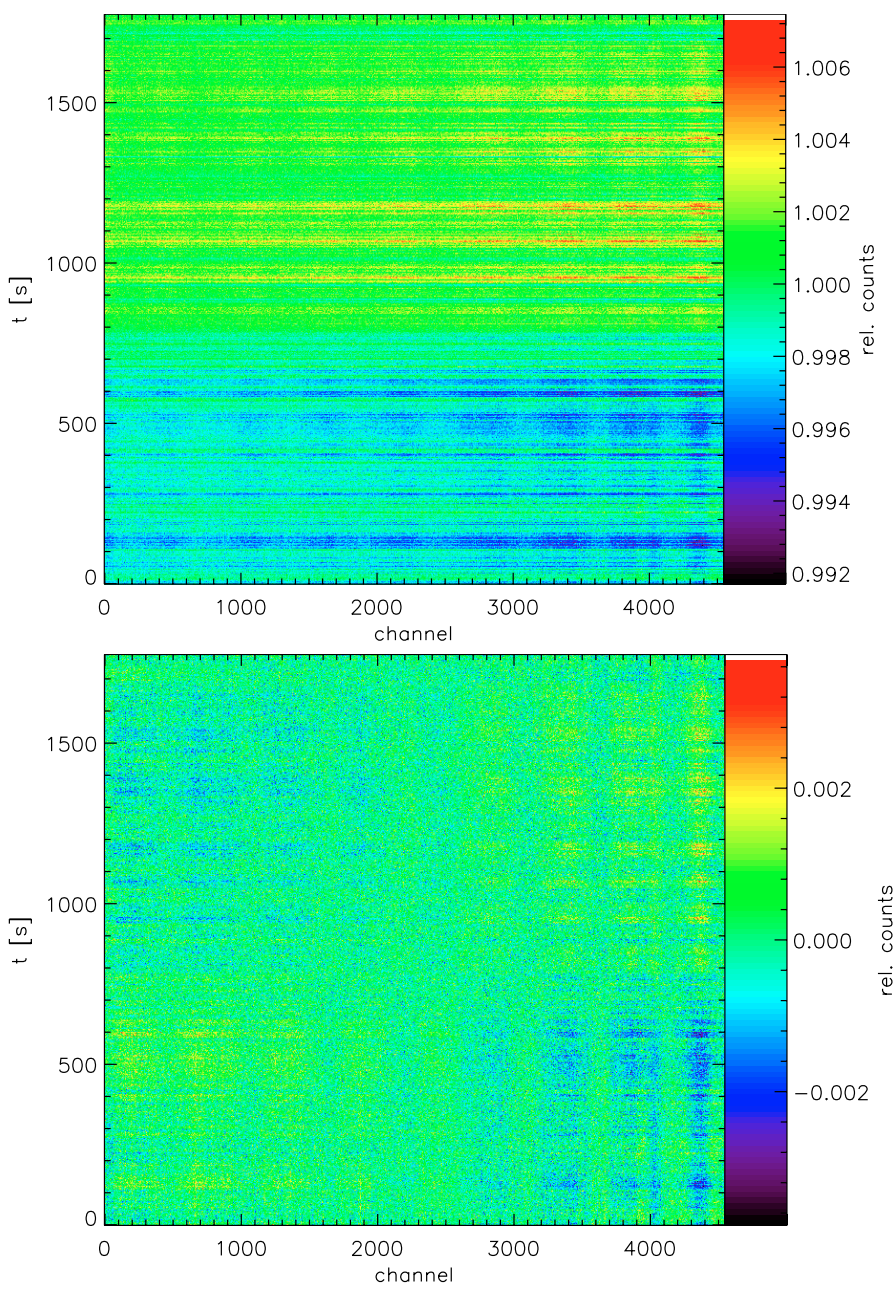

Fig. 1. Time series of spectrometer data where either the total-power normalisation (Eq. (1), upper plot) or the spectroscopic normalisation (Eq. (2), lower plot) is applied. The data were taken in stability measurements of the HIFI band $6 \mathrm{IF}$ chain in V-polarisation using the wide band spectrometer (WBS, Schieder et al. 2000) in June 2007. The three WBS subbands covering the available IF bandwidth are plotted sequentially, appearing as a single spectrometer with 4556 channels.

of two-channel Allan variance spectra taken over all reference channels $j$, except for the small contribution from the considered channel $i$ itself to the average which is not used in the twochannel spectroscopic Allan variance.

Figure 1 illustrates the two normalisations by plotting a time series of spectrometer data $s_{i}\left(t_{k}\right)$ both for the total power and the spectroscopic approach. Most variations are seen to occur equally in all channels so that they are mainly visible in the total power data. However, we also see some differential variations in terms of gain changes across the full band and variable standing wave features showing up as weak structures in the spectroscopically normalised plot.

HIFI and a number of other receivers cover a large bandwidth by combining several sub-spectrometers with a smaller bandwidth into one large array. In these array spectrometers, the different subbands will see partially different signal chains so that they may show different stability behaviours. Each subband has to be characterised individually. Thus all averages over the channels $i$ were computed only within the spectrometer subbands. In this way, we provide the stability numbers relevant for individual narrow lines, but we disregard the spectral purity of the full baseline. The latter may show steps between the different subbands due to dynamic platforming, i.e. a mutual drift of the gain between the subbands. To include these effects a second spectroscopic normalisation needs to be used where the average over all channels $i$ in Eq. (2) is computed for the full spectrometer. Examples are given in Sect. 4. In case of sufficient overlap between the subbands, dynamic platforming can be corrected in the data reduction process so that we can restrict ourselves to the stability analysis for the individual subbands.

Altogether, each stability measurement characterises two kinds of instabilities. When performing the analysis at the normalised data given by Eq. (1) we measure the total power stability. This result has to be used when setting up observations which aim for a determination of the continuum level, as is the case for measurements of absorption lines. With the spectroscopically normalised data from Eq. (2) we measure the spectroscopic stability, that can be used for observations which do not aim for an accurate determination of the continuum level, e.g. observations of molecular emission lines. The spectroscopic stability always exceeds the total power stability leading to more efficient observing schemes, so that one has to find a compromise between the need for an accurate continuum level determination and the request for a high observing efficiency.

\section{Computation of the Allan variance}

\subsection{Convolution schemes}

The computation of the Allan variance consists of a convolution of the signal data $s_{i}\left(t_{k}\right)$ from Eqs. (1) or (2) by a Haar wavelet of size $L$

$\square_{L}=\left\{\begin{array}{c}1 / L \text { for }-L \leq t<0 \\ -1 / L \text { for } 0 \leq t<L \\ 0 \quad \text { everywhere else }\end{array}\right.$

and the computation of the variance of the convolved signal ${ }^{1,2}$ :

$\sigma_{\mathrm{A}, i}^{2}(L)=\left\langle\left(s_{i}\left(t_{k}\right) * \square_{L}-\left\langle s_{i}\left(t_{k}\right) * \square_{L}\right\rangle_{k}\right)^{2}\right\rangle_{k}$

Plotting the Allan variance $\sigma_{\mathrm{A}}^{2}(L)$ as a function of the filter size $L$ shows the variation of the signal on the scale of the temporal lag $L$. Computing the Allan variance by actually convolving the time series by the $\square$-filter function for each time step can be considered a waste of computing time, because neighbouring values in the convolved time series are no longer statistically independent. Independent convolution results are only obtained for

\footnotetext{
${ }^{1}$ Note that the original definition of the Allan variance is smaller by a factor $1 / 2$. We omit this factor to allow a direct comparison to the drift error in observations.
}

2 The variance definition used here is

$\sigma^{2}=\left\langle\left(x_{k}-\left\langle x_{k}\right\rangle_{k}\right)^{2}\right\rangle_{k}=\frac{1}{N} \sum_{k=1}^{N}\left(x_{k}-\left\langle x_{k}\right\rangle_{k}\right)^{2}$.

This deviates from the ordinary variance definition

$\sigma_{\text {standard }}^{2}=\frac{1}{N-1} \sum_{k=1}^{N}\left(x_{k}-\left\langle x_{k}\right\rangle_{k}\right)^{2}$

for small numbers $N$. It has, however, the advantage that it is independent of the exact way of sampling a given continuous distribution, measuring only the internal properties of the distribution, as long as the sampling is dense enough. 
points separated by a lag larger than the filter size $L$. A more efficient method is thus to compute the convolution integral only for filter settings separated by the filter size (Schieder et al. 1985). When we chose filter length $L$ to be an integer multiple of the step size $\Delta t=t_{k+1}-t_{k}$, i.e. $L=l \times \Delta t$, we can use the average over a reduced number of points to compute the Allan variance

$\sigma_{\mathrm{A}, i}^{2}(L)=\left\langle\left(S_{i}(K)-S_{i}(K+1)-\left\langle S_{i}(K)-S_{i}(K+1)\right\rangle_{K}\right)^{2}\right\rangle_{K}$

with

$S_{i}(K)=\frac{1}{l} \sum_{k=K l+k_{1}}^{(K+1) l+k_{1}-1} s_{i}\left(t_{k}\right)$.

Compared to the full convolution (Eq. (4)) this corresponds to counting only points separated by $L$ in the convolved function $s_{i}\left(t_{k}\right) * \square_{L}$ when computing the variance. Each data point of the signal contributes twice - once in the positive and once in the negative term for the binned signal.

One disadvantage of this method is the loss of information from points at the beginning or the end of the time series which are not covered by full intervals of size $2 L$. The discrete sampling approach necessarily ignores an arbitrary fraction of the data at the beginning or end of the time series when the time series does do not match integer multiples of the filter size. This is obviously a small effect for small filters, but leads to a noticeable effect for large filters. Moreover, the selection of the start frame $k_{1}$ for the convolution is arbitrary. By shifting this through one filter length, different results are obtained for the Allan variance in case of a finite statistics in the time series of data. This is demonstrated in Fig. 2a. For each filter lag $L$ we have computed the result of Eq. (5) when shifting the start frame $k_{1}$ through the full filter length $2 L$. We find a large scatter in particular for large lags, i.e. a noticeable uncertainty of the result which depends on the arbitrary selection of this start frame. We can compare this with the result from the full convolution of the time series in Fig. 2b. The Allan variance from the full convolution provides a very smooth curve while the data from any particular $L$ sampling (shown in Fig. $2 \mathrm{~b}$ for $k_{1}=1$ ) show a considerable scatter around this curve. Nevertheless, almost all values fall within the statistical error bars computed from the finite size of the data series below.

We can conclude that the brute-force approach of the full convolution of the time series with the filter function provides the best results by not neglecting any data and by guaranteeing that every feature in the spectrum is covered by an appropriate filter setting. This results in smooth curves of the Allan variance spectra facilitating fitting and interpreting by the naked eye. Using today's computer technology, the convolution is practically possible by means of a Fast-Fourier transform for spectrometer time series of up to a few thousand steps. For longer time series, the numerically simpler approach of the discrete sampling can be used. The sampling in steps of $L$ still provides reliable data within the statistical uncertainty inherent to the Allan variance analysis. However, the noticeable error bars for large lags always have to be taken into account.

\subsection{Error estimate}

To compute the statistical uncertainty of the Allan variance we concentrate on this intrinsic uncertainty of the method neglecting the error propagation of possible uncertainties of the measured data into the Allan variance values.
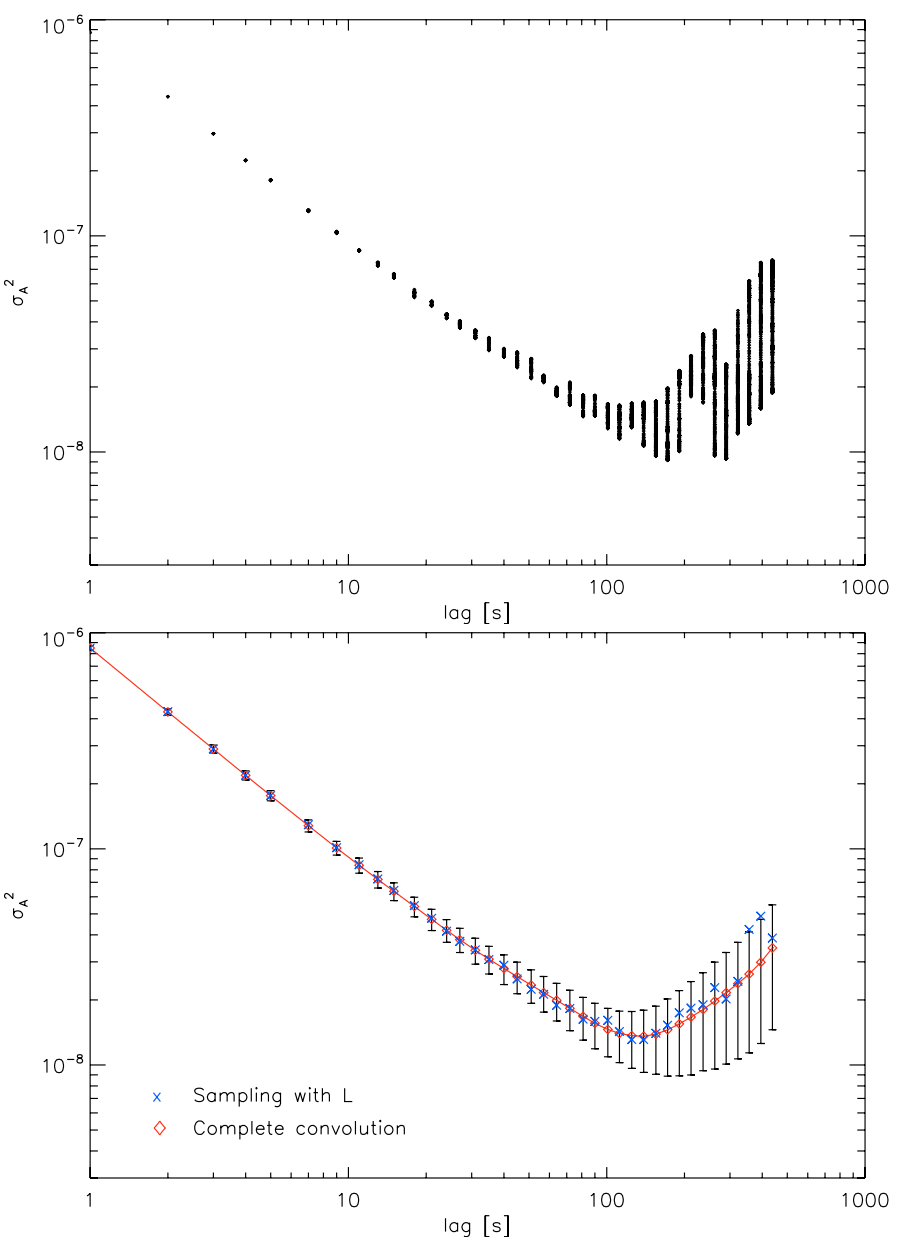

Fig. 2. Spectroscopic Allan variance spectrum computed by discrete sampling of the time series in steps of the lag $L$ for all possible starting points $l_{1}$ in the time series (upper plot) compared to the spectrum computed by full convolution of the time series with the $\square$-wavelet (lower plot). The crosses in the lower plot represent the result from the $L$-sampled computation for $k_{1}=1$. The error bars shown for the results from the full convolution integral are determined by the statistical uncertainty due to the finite number of data points (see Sect. 3.2).

The statistical error results from the sampling error when scanning a continuous distribution $f(t)$ by taking $N$ data values at discrete randomly selected points $K$. It is well known that the uncertainty in the determination of the average value of the sampled distribution is given by a Poisson counting error and the variance of the distribution

$\delta\langle f\rangle_{K}=\sqrt{\frac{\left\langle\left(f-\langle f\rangle_{t}\right)^{2}\right\rangle_{t}}{N}}$

Equivalently, one can derive the uncertainty of the measured variance of the distribution caused by the discrete sampling as

$$
\begin{aligned}
\delta\left\langle\left(f-\langle f\rangle_{K}\right)^{2}\right\rangle_{K} & =\sqrt{\frac{\left\langle\left(f-\langle f\rangle_{t}\right)^{4}\right\rangle_{t}-\left\langle\left(f-\langle f\rangle_{t}\right)^{2}\right\rangle_{t}^{2}}{N}} \\
& =\left\langle\left(f-\langle f\rangle_{t}\right)^{2}\right\rangle_{t} \sqrt{\frac{K u r-1}{N}}
\end{aligned}
$$

where Kur denotes the kurtosis of the distribution, characterising its fourth moment. Gaussian distributions exhibit a kurtosis value of 3. Exponential distributions show $K u r=6$. Thus the 
relative accuracy of the measured variance of a distribution depends mainly on the number of points used to sample the distribution. The kurtosis measuring the relative strength of the wings of the distribution weighs this counting error by a factor of a few.

It is obvious that these principles apply as well to the determination of the Allan variance. As discussed above, each distribution of filter-convolved data values can only be sampled in steps of $L$ to obtain statistically independent values. If the data represent a random series, sampling in uniform time steps corresponds to a random sampling of the distribution of data values so that the equation above can be applied. This assumption is not always fulfilled in the measurement of drift processes but it is in general justified when sufficiently long time series are measured. Thus we can estimate the statistical error of the Allan variance for a time series with the length $N \times L$ by

$\delta \sigma_{\mathrm{A}, \mathrm{i}}^{2}(L)=\sqrt{\frac{\left\langle Z_{i, k}^{4}(L)\right\rangle_{k}-\left\langle Z_{i, k}^{2}(L)\right\rangle_{k}^{2}}{N}}$

with

$Z_{i, k}(L)=s_{i}\left(t_{k}\right) * \square_{L}-\left\langle s_{i}\left(t_{k}\right) * \square_{L}\right\rangle_{k}$.

These error bars are plotted in Fig. 2. When comparing the plots in Fig. 2, it is remarkable that the scatter obtained while varying $k_{1}$ gives a very good match to the statistical uncertainty shown as error bars of the convolved data.

\subsection{How to characterise a full spectrometer?}

All computations so far were restricted to a time series of arbitrary data, i.e. an individual spectrometer channel $i$, but they ignored that a full instrument consists of thousands of spectrometer channels which are partially, but not completely independent.

The first approach to characterise the full spectrometer is the computation of the Allan variance channel by channel and the visualisation of the result in a three-dimensional plot. The result of such a channel-by channel analysis is demonstrated in the upper part of Fig. 3 showing the total-power Allan variance in logarithmic units for the HIFI test measurement from Fig. 1. Three subbands of the wide band spectrometer cover the full spectrum; their boundaries are visible at channels 860 and 2708. We clearly see that the instability of the system is dominated by a kind of standing-wave pattern superimposed on a large-scale trend of higher stabilities at lower frequencies (smaller channel numbers). We find a pattern of alternating regions of more and less stable channels. This can be interpreted in terms of the baseline of example spectra. The lower plot in Fig. 1 shows the difference of two spectra obtained by averaging over two adjacent $500 \mathrm{~s}$ intervals. We recognise a kind of standing-wave baseline distortion with a period matching the features visible in the Allan variance spectra. The amplitude of this baseline distortion grows towards higher channel numbers where we find larger values of the Allan variance at the upper boundary of the three-dimensional plot corresponding to $L \approx 500 \mathrm{~s}$.

From this plot it is obvious that variations of the instrumental behaviour across the spectrometer have to be considered. This provides the full information, but has the practical disadvantage that a surface plot is more difficult to interpret by eye than a two-dimensional plot, that it is not possible to include information about the error bars in the plot, and that for the optimisation of the observing strategy we have to reduce the result to a few numbers. To get a rough feeling for the spectral behaviour one
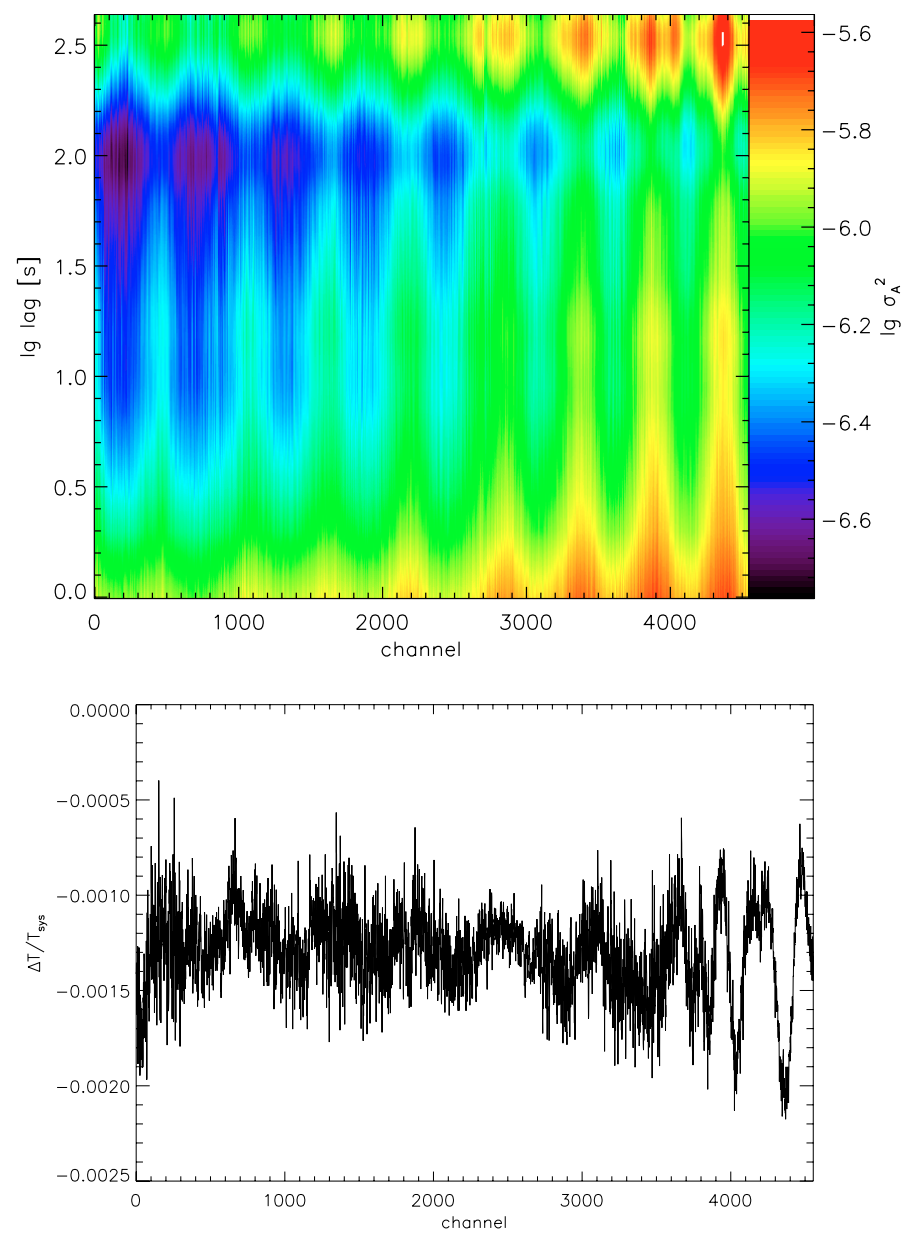

Fig. 3. Channel-by channel total-power Allan variance spectra for the test measurement described in Fig. 1 (upper plot). The colour coding shows the logarithm of the Allan variance. The lower plot shows the difference of two spectra obtained by averaging over two consecutive $500 \mathrm{~s}$ intervals.

can also look at the difference baseline plot, however, the corresponding interval has to be selected arbitrarily, missing most of the statistics of the measurement, so that a single baseline can never provide all the information contained in the channel-bychannel Allan variance plot.

For the derivation of constraints for the observing strategy it seems plausible to characterise the whole instrument by the properties of the worst, i.e. most unstable channel. This is the most reliable approach also applicable to observations where the measurement in all channels is equally important, e.g. in frequency surveys of rich emission spectra with hundreds of lines per spectrum. However, it does not take into account that in most observations the observed lines cover only a very small fraction of the whole spectrometer output whereas bad channels are typically concentrated towards the edges of the IF band. Then, an average Allan variance spectrum is more appropriate.

One can consider three different ways of averaging. When starting from the channel-by-channel Allan variance analysis an average Allan variance spectrum is given by

$\sigma_{\mathrm{A}}^{2}(L)=\left\langle\left\langle\left(s_{i}\left(t_{k}\right) * \square_{L}-\left\langle s_{i}\left(t_{k}\right) * \bigsqcup_{L}\right\rangle_{k}\right)^{2}\right\rangle_{k}\right\rangle_{i}$

This approach corresponds to averaging the Allan variance spectrum obtained from two channels following the method by Schieder et al. (1985) over all pairs of channels. 


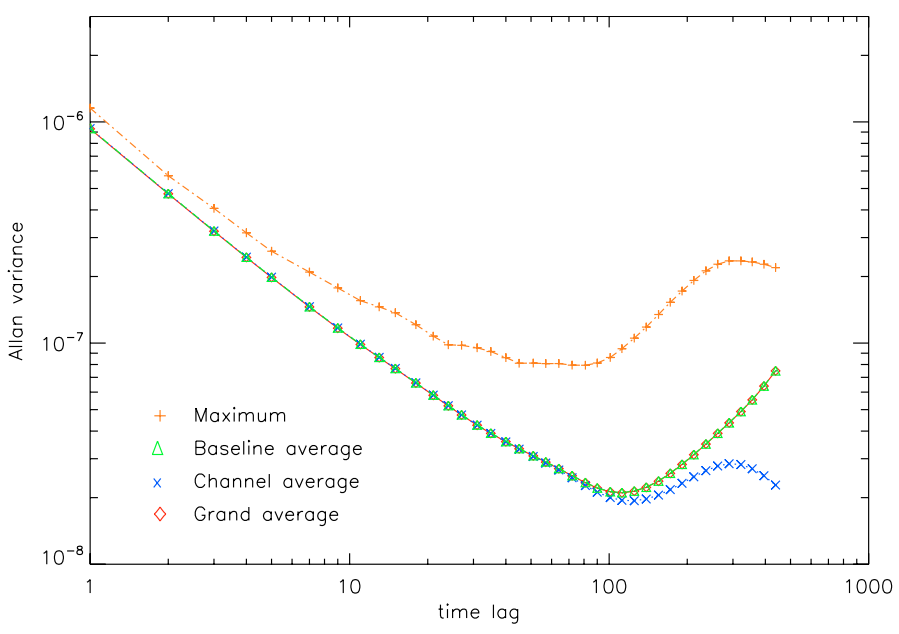

Fig. 4. Comparison of the four possible ways to characterise a full spectrometer band by a single Allan variance spectrum. Spectroscopically normalised data from the central subband shown in Fig. 1 have been used. The results when averaging the baseline Allan variance plots and when determining the variance relative to the grand average including simultaneously the channel scale and the time scale are so close that the curves are hardly distinguishable by eye.

A second possible approach is used by the baseline Allan variance method by Siebertz (1998). The variance within the convolved spectrum for each time step is considered and in a second step the average over all time steps is performed. We can write this as

$\sigma_{\mathrm{A}}^{2}(L)=\left\langle\left\langle\left(s_{i}\left(t_{k}\right) * \bigsqcup_{L}-\left\langle s_{i}\left(t_{k}\right) * \bigsqcup_{L}\right\rangle_{i}\right)^{2}\right\rangle_{i}\right\rangle_{k}$.

This kind of averaging is not able to monitor total-power variations because they enter the data $s_{i}\left(t_{k}\right) * \square_{L}$ in the same way as $\left\langle s_{i}\left(t_{k}\right) * \square_{L}\right\rangle_{i}$ so that they are subtracted and removed from the Allan variance spectrum. Therefore, the variance can only measure the spectroscopic Allan variance of an instrument.

The third option is to determine the variance relative to the grand average of the normalised and convolved spectra from the whole data field

$\sigma_{\mathrm{A}}^{2}(L)=\left\langle\left(s_{i}\left(t_{k}\right) * \square_{L}-\left\langle s_{i}\left(t_{k}\right) * \square_{L}\right\rangle_{k, i}\right)^{2}\right\rangle_{k, i}$.

The result for the spectroscopically normalised time series of the HIFI test measurement shown in Fig. 1 are demonstrated in Fig. 4. At all time lags below $100 \mathrm{~s}$ the different averages show identical Allan variance spectra with a slope of -1 characteristic for white radiometric noise. In contrast, the selection of the worst channel shows that this experiences some additional fluctuations even at the scale of $1 \mathrm{~s}$ and a drift noise growing to the amplitude of the radiometric noise already after $20 \mathrm{~s}$. Consequently, the relevant stability times are very short if one has to guarantee that the drift contribution remains small for each individual channel. The vast majority of all spectrometer channels behaves in a much more stable manner.

Comparing the average of the baseline Allan variance with the Allan variance using the grand average in the difference shows very similar spectra which are hardly to distinguish by eye, i.e. the variation of the convolved data relative to the average over the spectrum at a given time step is almost identical to the variation relative to the global average. In contrast, the average of the channel Allan variance spectra is always smaller at large

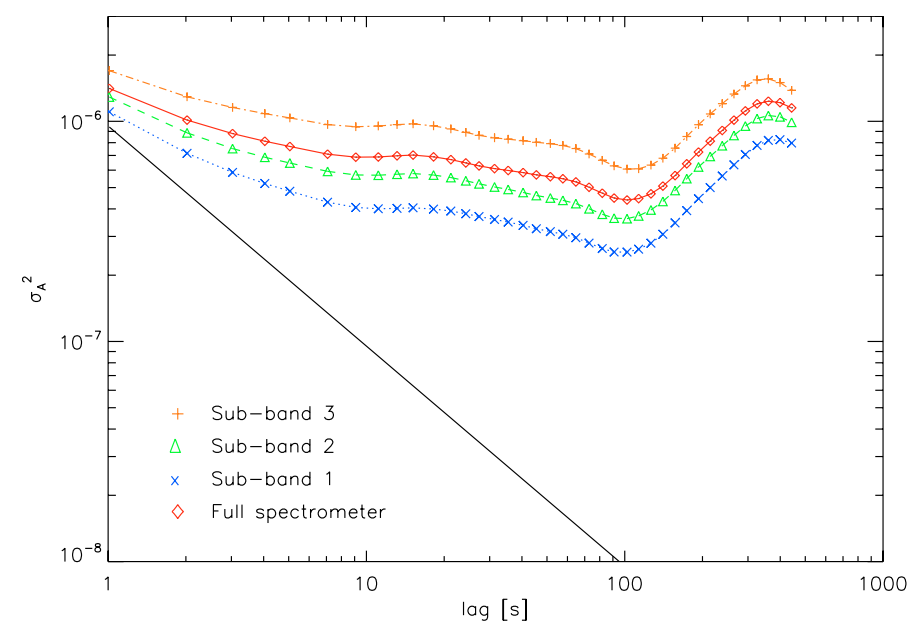

Fig. 5. Average total power Allan variance spectra for the different WBS subbands computed for the time series of data presented in Fig. 1.

lags, i.e. the variation of the convolved data relative to the average in the corresponding channel is always smaller than the variation relative to the global average. This can be understood from the the bandpass normalisation and the spectroscopic normalisation of the signal $s_{i}\left(t_{k}\right)$ discussed in Sect. 2.1. The bandpass normalisation guarantees that the average $\left\langle s_{i}\left(t_{k}\right)\right\rangle_{k}$ of any channel $i$ is identical to the global average $\left\langle s_{i}\left(t_{k}\right)\right\rangle_{k, i}$ whereas the spectroscopic normalisation leads guarantees the identity $\left\langle s_{i}\left(t_{k}\right)\right\rangle_{i}=$ $\left\langle s_{i}\left(t_{k}\right)\right\rangle_{k, i}$ for each time step. For spectroscopically normalised data, the convolution with the Allan filter hardly changes the average of the individual spectra, $\left\langle s_{i}\left(t_{k}\right) * \square_{L}\right\rangle_{i} \approx\left\langle s_{i}\left(t_{k}\right) * \square_{L}\right\rangle_{k, i}$ for each time step $i$, but for the individual channels, the convolution leads to offsets of $\left\langle s_{i}\left(t_{k}\right) * \bigsqcup_{L}\right\rangle_{k}$ from the global average when they have different trends which do not exactly cancel. For spectroscopically normalised data and sufficiently long time series in which all drifts do statistically cancel, all three averaging methods should provide the same results.

For practical application, the method should be chosen which is most adequate for the need of astronomical observations to control the drift error in measured data. Basically all spectroscopic observations are interested in the shape of a whole spectrum which is more than a bunch of independent channel data. Drift errors, showing up as irregular baseline distortions, can result in a clear degradation of the scientific value of the data even if the magnitude of the distortions does not exceed the radiometric noise in the data. To take the mutual relation of drift contributions across the spectrum into account we have to use the average of the baseline Allan variance or the Allan variance relative to the grand average. Because the baseline Allan variance is not able to characterise total power drifts we propose to always use the "grand average" method. It combines the advantage of the baseline Allan variance method reflecting the observer's view on spectroscopic data with the possibility of analyzing the drift behaviour of an instrument including total-power variations. However, we want to stress again that the characterisation of the instrument by a single Allan variance spectrum is only justified if an inspection of a plot like Fig. 3 has revealed that no strong deviations of the drift behaviour across the spectrometer occur.

An intermediate level of analysis can be provided for arrayspectrometers, like the HIFI-WBS, by characterising each spectrometer subband individually (see Sect. 2.2). Figure 5 shows the resulting total-power Allan variance spectra for the HIFI 
laboratory measurement already used for demonstration in Fig. 3. The solid line represents the limit of pure radiometric noise. The trend of channels of higher number (frequency) having lower stability (Fig. 3) is clearly seen. For the full spectrometer we obtain a kind of intermediate behaviour. Comparing the plot with Fig. 4 shows that the total-power Allan variance values suffer from much stronger drift contributions than the spectroscopically normalised values, with a noticeable drift already changed visible at very small lags. At all time lags below $100 \mathrm{~s}$, the spectrum shows a typical slope of about -0.3 . This is in agreement with stability measurements of the high electron mobility transistors used in the receiver amplifiers (Whyborn 2003), indicating that the total-power stability of the overall system is mainly determined by their gain fluctuations.

\section{Interpretation of Allan variance spectra}

\subsection{Comparison to radiometric noise}

The Allan variance always contains a combination of radiometric and drift fluctuations. They can be separated based on their different spectral characteristics. Fluctuations with a $f^{-\alpha}$ power spectrum show up in the Allan variance as $L^{\alpha-1}$ spectra (Schieder \& Kramer 2001). The superposition of white radiometric noise $(\alpha=0)$ and a power law drift noise with an arbitrary spectral exponent $\alpha$ gives an Allan variance spectrum

$\sigma_{\mathrm{A}}^{2}(L)=\frac{2}{B_{\mathrm{Fl}} L}+A L^{\alpha-1}$.

Here, $B_{\mathrm{Fl}}$ is the fluctuation bandwidth per backend channel determined by the power spectrum of the noise (Kraus 1980) and $A$ characterises the amplitude of the instrumental drift.

Subtracting the radiometric noise contribution, $2 /\left(B_{\mathrm{Fl}} L\right)$, from the Allan variance spectrum isolates the drift contributions. Moreover, it is useful to normalise the drift contribution by the radiometric noise because all observations will aim for data where the error due to instrumental drifts is small compared to the radiometric error of the observation and the radiometric noise can be easily calculated. We obtain the normalised drift Allan variance as

$\sigma_{\mathrm{A}, \mathrm{drift}}^{2}(L)=\frac{B_{\mathrm{Fl}} L}{2} \sigma_{A}^{2}(L)-1=\frac{B_{\mathrm{Fl}} A}{2} L^{\alpha}$.

This describes the actual impact of the drift noise for an astronomical observation, as is demonstrated in Fig. 6 for the spectroscopic channel-by-channel Allan variance of the data from Fig. 1. We find some regions where the drift contribution seems to be negative, indicated by the white contours. This is due to the fact that the fluctuation bandwidth is not completely constant across the spectrometer and when subtracting the radiometric noise for an average fluctuation bandwidth, we create slightly negative numbers for channels that have a fluctuation bandwidth that is eventually slightly larger than the average. The effect is always less than a few percent, so that we neglect it here. We recognise again the pattern of standing-wave like drift contributions, but the large scale gradient that was visible in the totalpower Allan variance from Fig. 3 is absent. The drift noise is strongly variable across the spectrometer. At $L=300 \mathrm{~s}$ it hardly exceeds the radiometric noise in the most stable regions while it exceeds it by a factor $>100$ in the most unstable regions of the spectrum.

We can use the knowledge of the shape of the radiometric noise contribution to derive the instrumental stability also from imperfect measurements not covering a continuous time series.

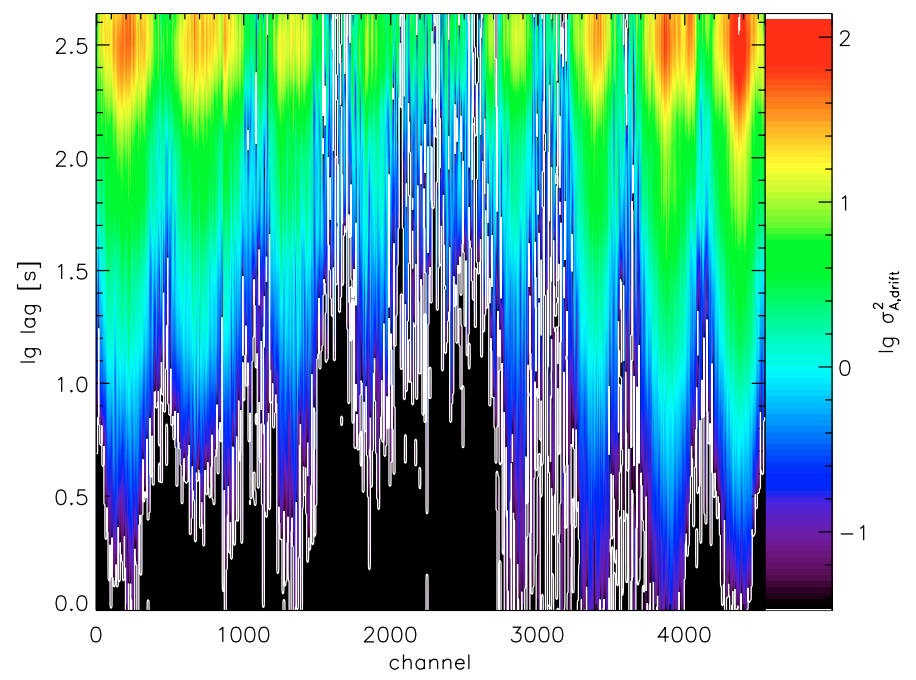

Fig. 6. Radiometrically normalized channel-by-channel spectroscopic drift Allan variance spectra, $\sigma_{\mathrm{A} \text {,drift }}^{2}(L)=B_{\mathrm{Fl}} L \sigma_{A}^{2}(L) / 2-1$, for the average fluctuation bandwidth of $B_{\mathrm{Fl}}=2.1 \mathrm{MHz}$. The color bar gives the logarithm of this variance. White contours indicate parts of the spectrum where the measured variations drop below the average radiometric noise level, i.e. where the normalized spectra fall below zero. The data from Fig. 1 are used so that the figure can be compared to the Allan variance plot in Fig. 3.

If the spectrometer readout adds a significant dead time between individual integrations, Eq. (14) does not hold any more because the radiometric noise is determined by the fraction of $L$ used for the integration, $L_{\mathrm{int}}=L-L_{\mathrm{dead}}$, while the drift is still determined by the total time lag between two measurements $L$. We can correct for these dead times as long as the Allan variance at the first lag $\sigma_{\mathrm{A}}^{2}\left(L_{1}, L_{\mathrm{int}, 1}\right)$ is still purely radiometric or if we know the level of the radiometric noise from independent computations. If we scale the radiometric contribution by the factor $L_{\text {int }} / L$, we obtain again a self-consistent Allan variance spectrum, depending only on the filter size $L$ :

$\sigma_{\mathrm{A}}^{2}(L)=\sigma_{\mathrm{A}}^{2}\left(L, L_{\text {int }}\right)-\sigma_{\mathrm{A}}^{2}\left(L_{1}, L_{\text {int }, 1}\right) \times L_{\text {dead }, 1} / L$.

This corrected spectrum can be used in the same way as the spectrum from perfect measurements to derive the Allan time.

\subsection{Definition of the stability time}

The Allan time is used to quantify the lag at which the Allan variance spectrums changes from being dominated by radiometric noise to being dominated by the instrumental drift. The traditional definition uses the minimum of the Allan variance spectrum. At smaller lags the fluctuations are dominated by the radiometric noise that drops with the integration time given by the Allan filter size, at larger lags the drift terms dominate resulting in an increase of the fluctuations with filter size. This approach, however, is only applicable if the drift follows a usual spectral characteristics with an index $\alpha>1$. Otherwise no minimum is formed. Figure 5 showed an example with an $L^{-0.3}$ dependence of the Allan variance corresponding to a $1 / f^{0.7}$ characteristics of the fluctuations and we have seen numerous other examples with a drift behaviour close to $1 / f$ noise leading to a very flat Allan variance spectrum.

The use of the Allan minimum time $t_{\mathrm{A}}$ thus has two disadvantages:

i) for drift noise shallower than $1 / f$ the Allan variance has no minimum although it is still a good measure for the stability of the system; 
ii) a small uncertainty in the spectral index of the drift function can lead to large shifts of the minimum making it very difficult to derive an accurate error estimate for the Allan minimum time from the statistical uncertainty of the Allan variance.

Therefore, we propose another definition of the Allan time $t_{\mathrm{A}}^{\prime}$ based on the normalisation discussed above: $t_{\mathrm{A}}^{\prime}$ is the lag for which the drift contribution to the total uncertainty equals the radiometric contribution, i.e. $\sigma_{\mathrm{A} \text {, drift }}^{2}\left(t_{\mathrm{A}}^{\prime}\right)=1$. This means that at $t_{\mathrm{A}}^{\prime}$ the total Allan variance amounts to twice the radiometric Allan variance. For a drift noise following a $1 / f^{2}$ spectral dependence, the new Allan time $t_{\mathrm{A}}^{\prime}$ agrees with the Allan minimum time $t_{\mathrm{A}}$.

The new Allan time definition has two practical disadvantages compared to the Allan minimum time:

i) it is easier to determine the minimum of the Allan variance by the naked eye than determining the point where it deviates by a factor 2 from the radiometric line;

ii) because most existing measurements characterise the instrument stability by the Allan minimum time, a comparison with their results requires an additional translation step. The relation between both Allan times can be computed from Eq. (14) as

$t_{\mathrm{A}}^{\prime}=(\alpha-1)^{1 / \alpha} t_{\mathrm{A}}$.

However, we can easily compute the uncertainty of the new Allan time $t_{\mathrm{A}}^{\prime}$ using the uncertainty of the Allan variance (Eq. (9)) by

$\frac{\delta t_{\mathrm{A}}^{\prime}}{t_{\mathrm{A}}^{\prime}}=\frac{1}{|\alpha|} \frac{\delta \sigma_{\mathrm{A}}^{2}\left(t_{\mathrm{A}}^{\prime}\right)}{\sigma_{\mathrm{A}}^{2}\left(t_{\mathrm{A}}^{\prime}\right)}$.

This error remains finite even in case of $1 / f$ noise whereas it diverges for the traditional Allan minimum time.

\subsection{Binning of spectra}

In astronomical data analysis it is common to use the average of several channels of a spectrometer to reduce the observational noise if the fixed resolution of the spectrometer is higher than the resolution needed to deduce physical parameters from the observed spectra. An example are observations of the [CII] line at 1.9 THz with the HIFI-WBS in which the astronomer asks for a frequency resolution of about $1 \mathrm{~km} / \mathrm{s}$, corresponding to $6.3 \mathrm{MHz}$ while the native spectrometer resolution is $1.1 \mathrm{MHz}$. It is a common misconception to assume that the noise in the spectra will drop by a factor $\sqrt{1 / 11}$ if the 11 channels are co-added that provide the effective resolution of $6.3 \mathrm{MHz}$.

For a correct treatment, the correlation between neighbouring channels has to be taken into account. The spectral correlation of the noise leads to a fluctuation bandwidth that differs from the channel spacing. If the autocorrelation function (ACF) of the spectrometer is known, the effective fluctuation bandwidth can be computed as a function of the binning width $n_{\text {bin }}$, following the formalism provided in Appendix A of Schieder \& Kramer (2001),

$B_{\mathrm{Fl}}\left(n_{\text {bin }}\right)=n_{\text {bin }} \Delta v_{\text {channel }} \times \frac{1+2 \sum_{m=1}^{\infty} g_{m}}{1+2 \sum_{m=1}^{n_{\text {bin }}-1}\left[1-m /\left(n_{\text {bin }}-1\right)\right] g_{m}}$

where $\Delta v_{\text {channel }}$ denotes the channel spacing and $g_{m}$ represent the values of the ACF at discrete channel shifts $m$. One can see that the native fluctuation bandwidth of the spectrometer $\left(n_{\text {bin }}=1\right)$

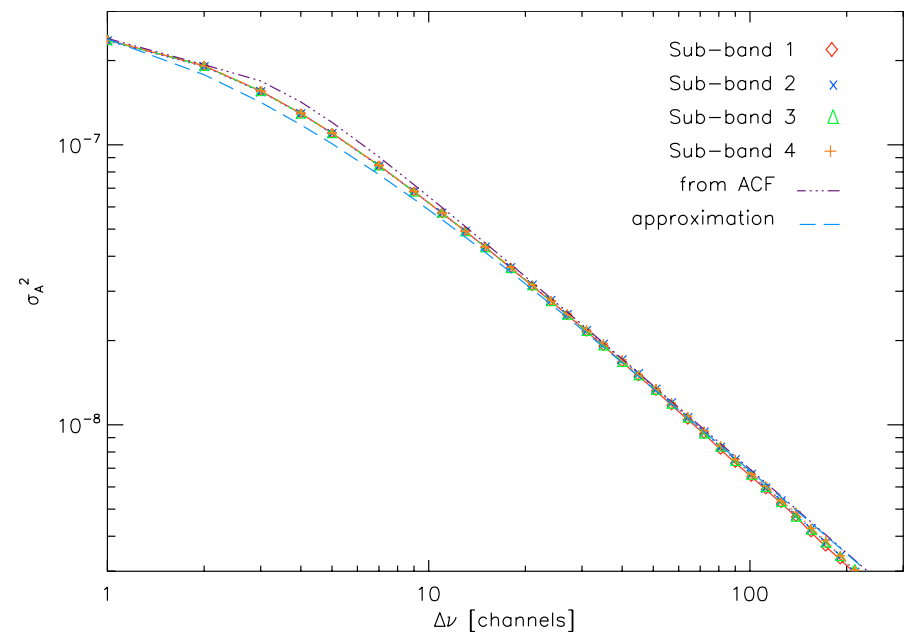

Fig. 7. Spectroscopic Allan variance at $L=5 \mathrm{~s}$ for stability measurements of the HIFI WBS (V-polarisation) as a function of the channel binning width. The difference between the four WBS subbands is too small to be visible by eye. The dash-dotted line shows the theoretical curve computed from the measured autocorrelation function following Schieder \& Kramer (2001). The dashed line represents the simple approximation with an additive constant.

always exceeds the channel spacing by the symmetrically integrated autocorrelation function, for the HIFI WBS this is a factor of almost three. The ACF has typically only a few non-vanishing coefficients $g_{m}$, so that for large values of $n_{\text {bin }}$ (large total binning widths) $B_{\mathrm{Fl}}$ approaches $n_{\text {bin }} \Delta v_{\text {channel }}$. Consequently, binning does not reduce the radiometric noise like $\sqrt{1 / n_{\text {bin }}}$ relative to the noise at the native resolution, but in the limiting case of large bin widths the noise is still about a factor $\approx \sqrt{3}$ higher.

In Fig. 7 we show this behaviour by computing the Allan variance for short lags in a stability measurement of the HIFI wide band spectrometer when applying a varying channel binning to the data. The dash-dotted line represents the curve given by Eq. (19) using laboratory data for the ACF measured by tracing a tunable narrow line source. We find a good agreement between the theoretical curve and the measured Allan variance data showing that we deal with pure radiometric noise at short lags, but a deviation by up to $10 \%$ at $n_{\text {bin }} \approx 3 \ldots 7$. The reason for this deviation is not clear. An imperfect line source used when measuring the ACF might lead to an overestimate of $g_{m}$ for higher $m$ at cost of $g_{1}$, resulting in this kind of deviation. Taking the typical error bars of the Allan variance (Sect. 3.2) and the natural variation of the fluctuation bandwidth across a spectrometer (Sect. 4.1) into account, this small deviation can be completely neglected in the following.

To quantitatively describe the observed behaviour of the fluctuation bandwidth it is possible to use an even simpler approximation consisting just of a linear dependence and an additive constant for the native fluctuation bandwidth:

$B_{\mathrm{Fl}}\left(n_{\text {bin }}\right)=B_{\mathrm{Fl}}(1)+\left(n_{\text {bin }}-1\right) \Delta v_{\text {channel }}$.

This is shown as the dashed line in Fig. 7. We find again a small deviation at intermediate bin sizes, but an overall reasonable agreement, so that we can use this approximation for fast computations.

With known fluctuation bandwidth for a particular channel binning we can compute the corresponding Allan time by determining the ratio between drift noise and reduced radiometric noise. It is usually assumed (Schieder \& Kramer 2001; Kooi et al. 2006) that the binning only reduces the radiometric 

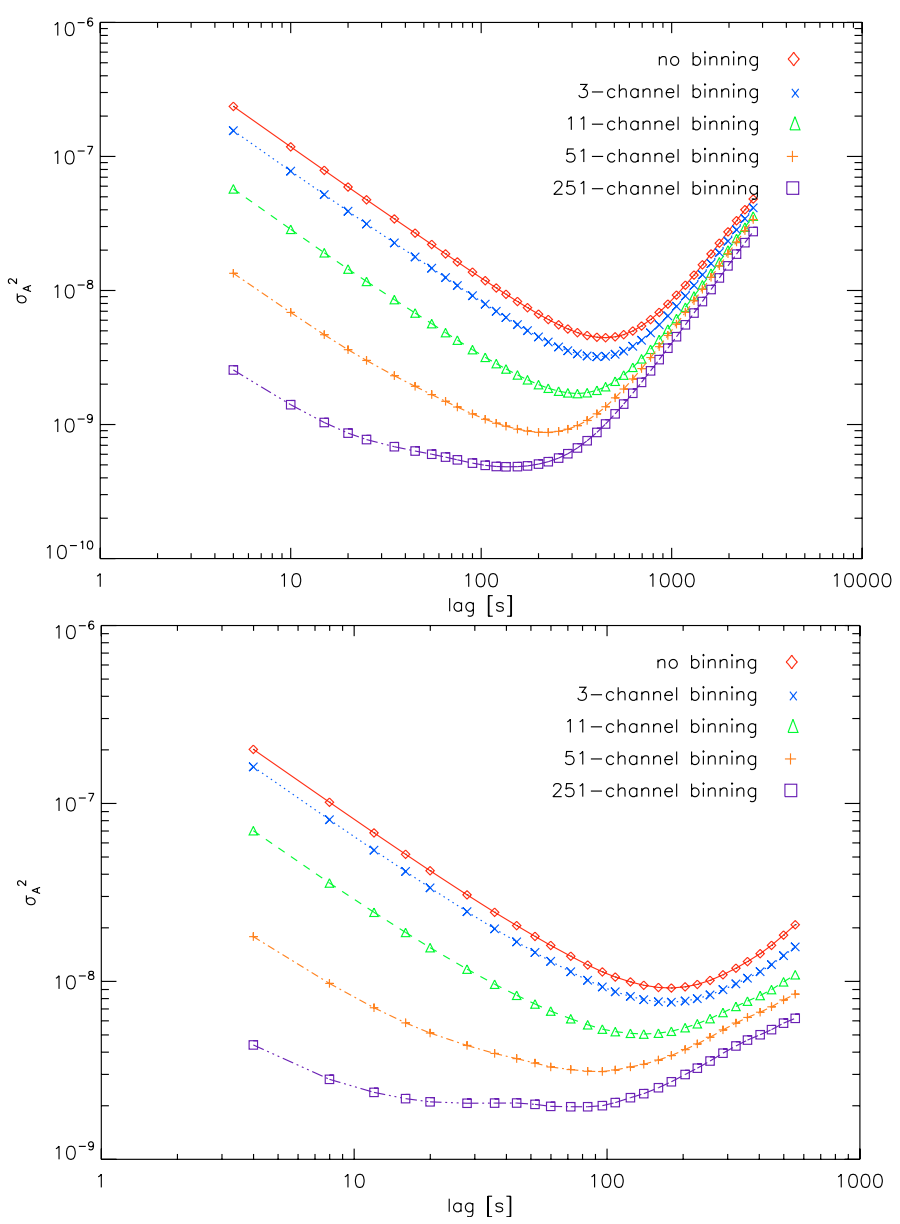

Fig. 8. Spectroscopic Allan variance spectra characterising the stability of subband 2 of the HIFI wide band spectrometer using a stable noise source (upper plot) and using the whole system (HIFI band 3a, lower plot) for five different levels of channel binning.

noise while the instrumental drift itself is not affected. Resolving Eq. (14) for $t_{\mathrm{A}}^{\prime}$ gives

$t_{\mathrm{A}}^{\prime}=\left(\frac{2}{A B_{\mathrm{Fl}}}\right)^{1 / \alpha}$

showing that the Allan time shifts to smaller lags when increasing the fluctuation bandwidth by

$t_{\mathrm{A}}^{\prime}\left(n_{\mathrm{bin}}\right)=\left(\frac{B_{\mathrm{Fl}}(1)}{B_{\mathrm{Fl}}\left(n_{\mathrm{bin}}\right)}\right)^{1 / \alpha} t_{\mathrm{A}}^{\prime}(1)$.

This relation is identical to the corresponding relation for the traditional Allan minimum time (Schieder \& Kramer 2001).

Figure 8 shows the impact of binning on the Allan variance spectra for data taken in a stability test of the spectrometer (upper plot) and for a test of the full system including mixer, local oscillator, and IF amplifier chain (lower plot). We show the spectroscopic Allan variance spectra for four different binnings and the native resolution. The data characterising the spectrometer alone perfectly follow the theoretical assumption of a constant drift contribution. Changing the binning width only changes the radiometric noise so that Eq. (22) applies.

For the whole system, however, we find a significant change of the drift contribution with the binning width, indicating that

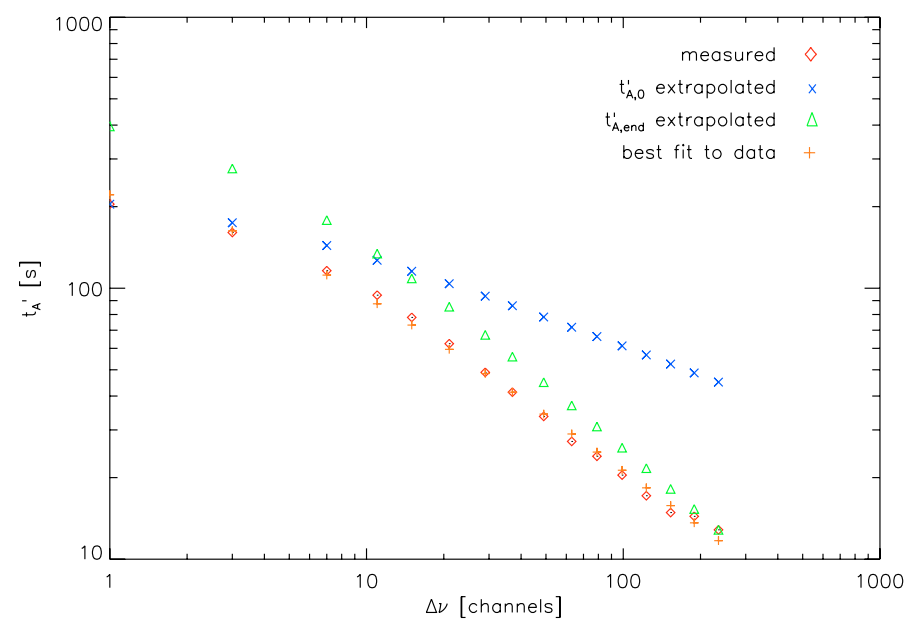

Fig. 9. Allan stability time $t_{\mathrm{A}}^{\prime}$ as a function of binning width for the stability measurements shown in the lower plot of Fig. 8. The red diamonds show the values measured from the individual Allan variance spectra. The blue crosses and green triangles show the theoretically expected behaviour for constant drift contributions using $t_{\mathrm{A}}^{\prime}$ and $\alpha$ from the original (non-binned) data or from the data with the largest binning width. The best fit to the measured data gives $t_{\mathrm{A}}^{\prime}=220 \mathrm{~s}$ and $\alpha=1.4$.

the assumption of a constant drift term is in general not applicable. We notice two effects:

i) the system is affected by some spectrally correlated fluctuations, like the unstable standing wave patterns discussed before, which are reduced by spectral binning. This leads to a reduction of the drift term and consequently to an increase of $t_{\mathrm{A}}^{\prime}\left(n_{\text {bin }}\right)$ relative to the value predicted by Eq. (22);

ii) the overall system is always affected by some $1 / f$ noise. When steeper drift noise and the radiometric contribution are sufficiently reduced by binning, the $1 / f$ noise starts to dominate the Allan variance. This reduces the Allan time relative to the value predicted by Eq. (22).

Altogether, this makes Eq. (22) questionable. Analysing the system tests of the HIFI instrument in different configurations we noticed deviations in both directions, cases with strong shallow noise, cases with narrow standing waves easily suppressed by binning, and cases with a complete cancellation, leading to an Allan time independent of the binning width. Finally we always performed the Allan variance analysis for a number of binning widths. The result for the HIFI band 3 a measurements used in Fig. 8 is shown in Fig. 9. Together with the measured values of $t_{\mathrm{A}}^{\prime}\left(n_{\text {bin }}\right)$ we show the values that would be obtained from Eq. (22) using the Allan time and spectral drift coefficient $\alpha$ measured for the first point (no binning) and the last point $\left(n_{\text {bin }}=235\right)$ in the plot. Both extrapolations overestimate the Allan stability time at other binning widths. We can, however, obtain a fit to the data using Eq. (22) by combining the information from all binning widths. This is shown as violet crosses which provide a very good match to the measured values. By using two different values for the exponent $\alpha$, one for the drift slope at a particular binning width and one to characterise the change of $t_{\mathrm{A}}^{\prime}$ with the binning size, we can thus provide a full characterisation of the instrumental stability with an accuracy of typically some ten percent.

\section{Optimisation of the observations}

Schieder \& Kramer (2001) used the Allan-variance minimum to derive constraints for the optimum timing of astronomical 
observations. They considered drift contributions with the two spectral indices $\alpha=2,3$ bracketing the behaviour often observed in spectroscopic Allan variance measurements. In the analysis of numerous HIFI stability measurements, we noticed, however, that a major fraction of total-power Allan variance spectra and still a significant portion of spectroscopic Allan variance spectra is dominated by shallower noise spectra, often close to $1 / f$ noise (see e.g. Fig. 5). Thus we repeat their computation for the general case of arbitrary noise spectral indices, also applying the new Allan time definition discussed above. Corresponding equations for the Allan minimum time were also derived by Schieder (priv. comm.).

Many observing modes are symmetric in the sense that equal time intervals are spent on the astronomical source and on the reference. These are beam-switch observations, moving either the telescope or a fast chopping mirror between source and reference, and frequency-switch observations. To compensate linear drifts, the most appropriate observing scheme consists of reference-source-source-reference sequences, with an integration time $t_{\mathrm{s}}$ for each source phase, the same time $t_{\mathrm{s}}$ in the reference phases, and a dead time $t_{\mathrm{d}}$ in between.

By using the information about the instrumental drift obtained from the Allan variance spectrum, in particular the Allan time $t_{\mathrm{A}}^{\prime}$ and the spectral index of the drift $\alpha$ it is possible to compute the average drift error in each difference measurement $S_{k}-R_{k}$, where $S_{k}$ is the integrated signal over the $k$ th source phase and $R_{k}$ is the integrated signal during the $k$ th reference phase. The total uncertainty of the astronomical measurement is then characterised by the variance

$\sigma_{\mathrm{obs}}^{2}\left(t_{\mathrm{s}}, t_{\mathrm{d}}\right)=\left\langle\left(S_{k}-R_{k}-\left\langle S_{k}-R_{k}\right\rangle_{k}\right)^{2}\right\rangle_{k}$

Following the formalism provided by Schieder \& Kramer (2001) we can derive this variance normalised to the average signal level from Eq. (14)

$$
\begin{aligned}
\frac{\sigma_{\mathrm{obs}}^{2}\left(t_{\mathrm{s}}, t_{\mathrm{d}}\right)}{\langle s\rangle^{2}}= & \frac{2}{B_{\mathrm{F} 1} t_{\mathrm{s}}} \\
& +A \frac{\left(2 t_{\mathrm{s}}+t_{\mathrm{d}}\right)^{\alpha+1}-2\left(t_{\mathrm{s}}+t_{\mathrm{d}}\right)^{\alpha+1}+t_{\mathrm{d}}^{\alpha+1}-2 t_{\mathrm{s}}^{\alpha+1}}{2\left(2^{\alpha}-2\right) t_{\mathrm{s}}^{2}} .
\end{aligned}
$$

The first term describes the radiometric noise which does not depend on the dead time. The second term is the drift noise. For $t_{\mathrm{d}}=0$ we return to the known relation (14) for the Allan variance with no dead time between subsequent data dumps. Equation (25) holds for all spectral indices $\alpha$ between 0 and 3 except for $\alpha=1$ where a logarithmic divergence occurs leading to a somewhat different functional description.

Substituting $A$ by $t_{\mathrm{A}}^{\prime}$ using Eq. (21) and normalising all times relative to the Allan time gives

$$
\begin{aligned}
\frac{\sigma_{\mathrm{obs}}^{2}(x, d)}{\langle s\rangle^{2}}= & \frac{2}{B_{\mathrm{Fl}} t_{\mathrm{A}}^{\prime}} \\
& \times\left(\frac{1}{x}+\frac{(2 x+d)^{\alpha+1}-2(x+d)^{\alpha+1}+d^{\alpha+1}-2 x^{\alpha+1}}{2\left(2^{\alpha}-2\right) x^{2}}\right)
\end{aligned}
$$

with $x=t_{\mathrm{s}} / t_{\mathrm{A}}^{\prime}$ and $d=t_{\mathrm{d}} / t_{\mathrm{A}}^{\prime}$. The use of the traditional Allan minimum time in this equation would add a factor $1 /(\alpha-1)$ to the drift term. For $\alpha=2$ and $\alpha=3$ one can then reproduce the results obtained by Schieder \& Kramer (2001).

The optimum observing mode is characterised by a minimum total noise, composed of radiometric and drift noise, obtained in a given observing time $t_{\mathrm{tot}}$. Summing up the $t_{\mathrm{tot}} /\left(2 t_{\mathrm{S}}+t_{\mathrm{d}}\right)$

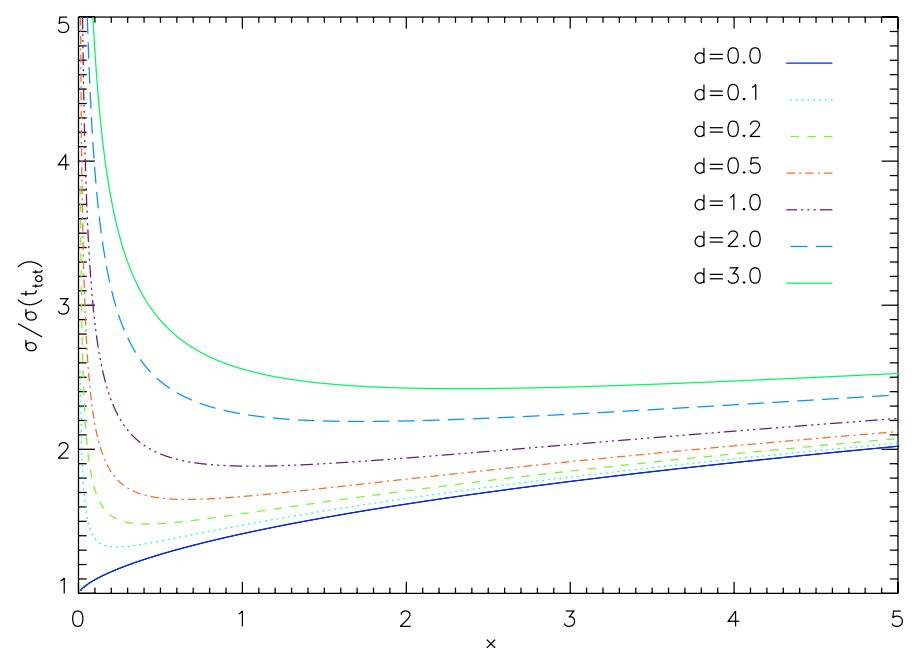

Fig. 10. Total noise in beam-switch observations consisting of radiometric and drift contributions relative to the radiometric noise from an ideal instrument as a function of the relative chop phase length $x$. The different curves represent different relative dead time per cycle. A spectral index $\alpha=0.7$ is used for the drift noise.

source-reference pairs fitting in the total observing time, we obtain the total noise of the observation as

$$
\begin{aligned}
\frac{\sigma_{\mathrm{tot}}^{2}(x, d)}{\langle s\rangle^{2}}= & \frac{4 x+2 d}{B_{\mathrm{Fl}} t_{\mathrm{tot}}} \\
& \times\left(\frac{1}{x}+\frac{(2 x+d)^{\alpha+1}-2(x+d)^{\alpha+1}+d^{\alpha+1}-2 x^{\alpha+1}}{2\left(2^{\alpha}-2\right) x^{2}}\right)
\end{aligned}
$$

(see Eq. (7)). The behaviour of this total noise is shown in Fig. 10 for a drift spectral index of 0.7 corresponding to the total-power measurements demonstrated in Sect. 4.1. We plot the standard deviation of the noise relative to the radiometric noise of an ideal instrument having no dead times, i.e. relative to $\sigma_{\text {ideal }} /\langle s\rangle=2 / \sqrt{B_{\mathrm{Fl}} t_{\mathrm{tot}}}$, as a function of the integration time per cycle relative to the Allan time $t_{\mathrm{A}}^{\prime}$ for different relative dead times. We find the same general shape of the curve as shown by Schieder \& Kramer (2001) for a drift spectral index of 2, with a high noise for short integration times due to the overhead from the dead time, an elevated noise for large integration times due to the instrumental drift and a minimum defining the optimum integration time. However, for the shallow drift index, the minima are much wider than the corresponding minima computed by Schieder \& Kramer (2001).

We can use this plot to compute optimum cycle times from $t_{\mathrm{A}}^{\prime}$ in this case, even if the Allan variance spectrum shows no minimum. We obtain the optimum integration time per cycle as a function of the instrumental dead time and the spectral index of the drift contributions by

$$
\begin{aligned}
x_{\mathrm{opt}}=t_{\mathrm{A}, \Delta v} \operatorname{root}_{x}\{ & (2 x+d)^{\alpha+1}(\alpha x-d)+(x+d)^{\alpha+1} d \\
& -2(x+d)^{\alpha+1}(2 x+d)(\alpha x+d)-d^{\alpha+1}(x+d) \\
& \left.-x^{\alpha+1}[\alpha(2 x+d)-d]-\left(2^{\alpha}-2\right) x d\right\}
\end{aligned}
$$

where $\operatorname{root}_{x}\{\}$ denotes the solution of the expression with respect to $x$.

Figure 11 shows this optimum integration time providing the minimum total noise as a function of the dead time per cycle for a set of different spectral drift indices $\alpha$. For spectral indices between 1.5 and 3 the curves hardly depend on the exact value of $\alpha$. When the dead time exceeds half the Allan time the optimum 


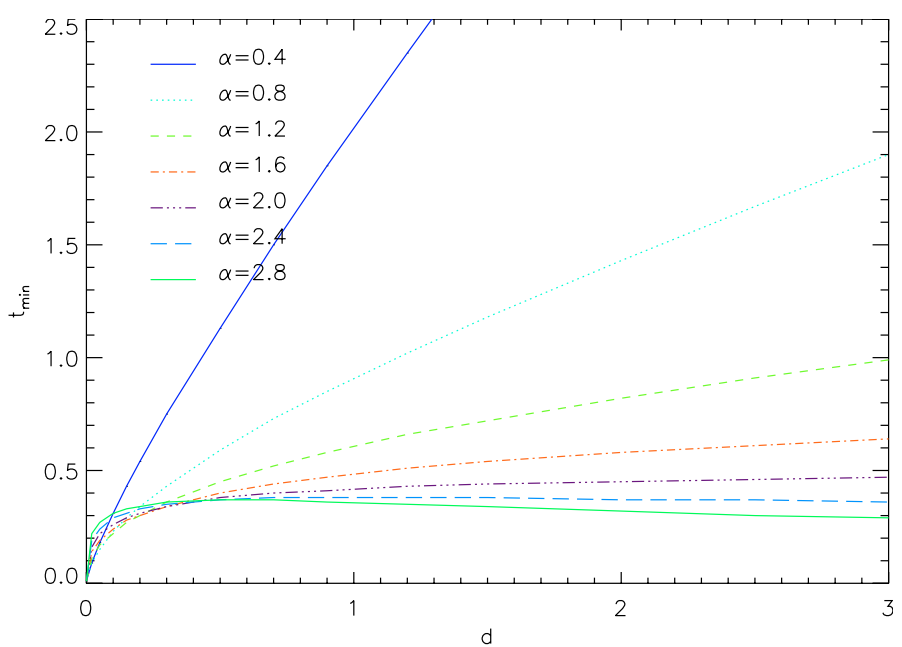

Fig. 11. The optimum phase length as a function of the relative overhead from the dead time, $d=t_{\mathrm{d}} / t_{\mathrm{A}}^{\prime}$, for different spectral indices $\alpha$ of the instrumental drift.

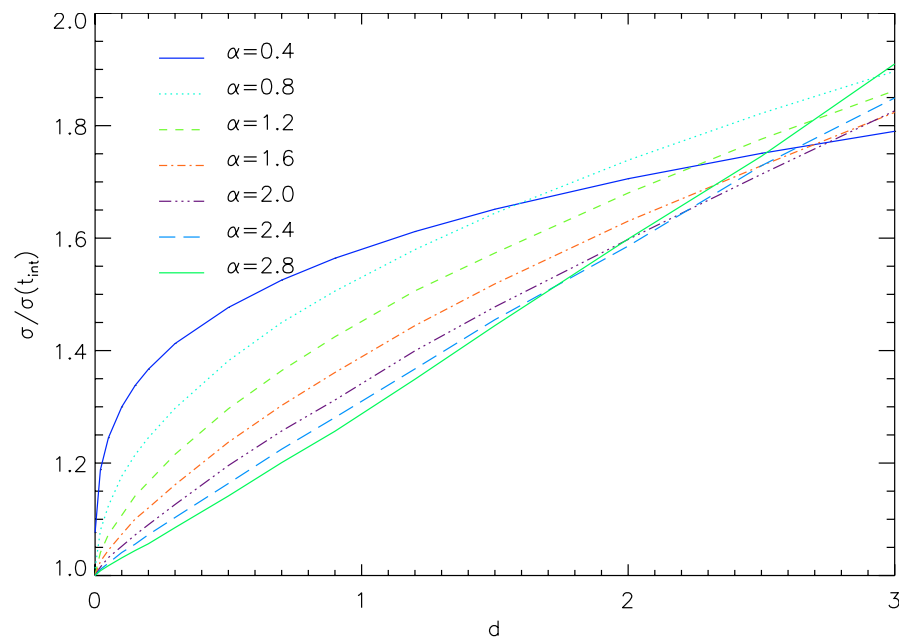

Fig. 12. Total noise relative to the radiometric noise for the optimum phase length as a function of the relative dead time per cycle, $d=t_{\mathrm{d}} / t_{\mathrm{A}}^{\prime}$, for different spectral indices $\alpha$ of the instrumental drift.

integration time saturates also at about half the Allan time. For shallow fluctuation spectra, however, the optimum integration time increases rapidly with the dead time so that it can easily exceed the Allan time for long dead times and spectral indices $\alpha<1$.

This effect somewhat relaxes the constraints for planning observations aimed at an accurate measurement of the continuum level. Although the total-power Allan time $t_{\mathrm{A}}^{\prime}$ is usually very short, the corresponding spectral index of the fluctuations it is often very shallow, $\alpha \approx 1$, allowing cycle times which exceed the Allan time so that the frequency for switching between source and reference can be lower than the pure Allan time suggests.

If the observations are set up with an optimum cycle length we can use Eq. (27) to compare the expected drift noise from an observation with the radiometric noise. This ratio is essential for the observer because it characterises the amplitude of systematic baseline distortions due to instrumental fluctuations relative to the white noise in the spectra. It is a measure of the size of possible baseline ripples which might be visible in the noise, and thus directly determine the quality of the observations. Figure 12 shows the total noise relative to the radiometric contribution for the optimum cycle length, i.e. $\sigma_{\text {tot, opt }} /\langle s\rangle \times \sqrt{B_{\mathrm{Fl}} t_{\mathrm{tot}} /(4+2 d / x)}$. In case of shallow drift spectra we notice a major drift

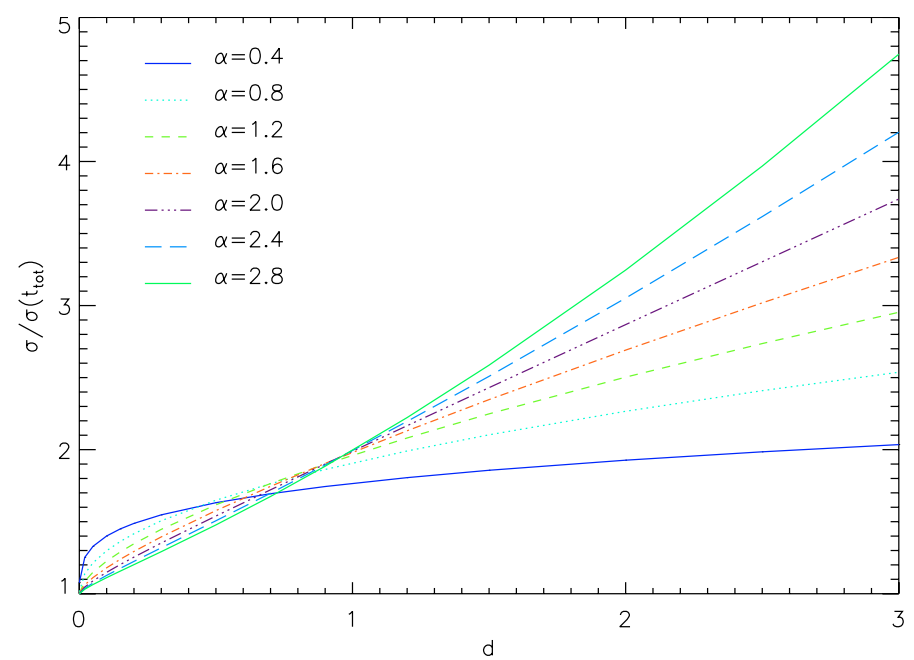

Fig. 13. Total noise RMS in a switched observation relative to the radiometric noise from an ideal instrument, $\sigma_{\text {tot opt }} \times \sqrt{B_{\mathrm{Fl}} t_{\mathrm{tot}}} /(2\langle s\rangle)$, for the optimum phase length as a function of the relative dead time per cycle.

contribution already for relatively small dead times whereas steep fluctuation spectra result in an almost linear growth of the drift contribution with dead time. However, as long as the dead time is smaller than about three Allan times, the total noise is increased by less than a factor 2, i.e. the drift contribution can still be hidden in the radiometric noise of the baseline.

Finally we can use Eq. (27) in astronomical time estimates, to provide the user with realistic numbers for the total data uncertainty obtainable in a given observing time. Figure 13 shows the total noise relative to the radiometric noise from an ideal instrument, $2 / \sqrt{B_{\mathrm{Fl}} t_{\mathrm{tot}}}$, for the optimum cycle as a function of the spectral index of the instrumental drift and relative dead time. For dead times exceeding about half the Allan time the drift contribution has the smallest impact for very shallow noise spectra whereas steep spectra naturally create drift contributions that grow quickly in time. The figure gives a direct measure for the efficiency gain that can be obtained by constructing a faster chopper mechanism or a more rapidly moving telescope to minimise the dead time relative to the Allan time.

We can apply exactly the same approach to determine the timing in second order observing loops set up to correct residuals from the simple differencing measurements. A typical example is a dual beam-switch measurement where a chopping mirror switches quickly between source and reference, but where the difference spectrum contains some standing wave residuals due to the different optical paths between the two mirror positions. In this situation, a second loop is used to periodically move the whole telescope so that the assignment of the two mirror positions to source and reference is reversed. Adding the differences from the two telescope positions then removes the baseline residual while maintaining the signal. To determine the period for the telescope motions, we have to consider the stability of the baseline ripple, i.e. the stability of the difference spectra. We call this second-order or differential stability, because first order instabilities will be removed already by the fast reference cycles. All computations used so far can be applied in the same way to determine the period of the second-order loop if the Allan variance analysis is not applied to continuous measurements, but to differential measurements switching between source and reference where the normalised difference spectrum is used for $s_{i}\left(t_{k}\right)$. However, it is clear that the switching will introduce some dead times, so that the radiometric contribution has to be corrected following Eq. (16). In our HIFI tests, we found a very good 
second order stability for dual-beam switch measurements under most conditions, so that we could often only derive lower limits for the differential Allan time. The second-order stability is considerably worse for frequency-switch measurements following the same scheme.

The formalism described above has been implemented in HSPOT, the Herschel observation planning tool, for the HIFI instrument guaranteeing an optimum setup of the observations and providing a realistic estimate for the quality of the data that can be obtained in a given observing time. We have to stress, however, that it is applicable in the same way for ground basedtelescopes provided that stability measurements are performed for typical atmospheric conditions to obtain estimates for the Allan time and the drift exponent.

\section{Conclusions}

\subsection{The optimum method}

We propose a new scheme for the computation of the Allan variance of a time series of spectrometer data. It combines the advantages of the spectroscopic Allan variance by Schieder et al. (1985) with the advantages of the baseline Allan variance by Siebertz (1998) so that the same formalism can be used to analyse the stability of an instrument with respect to total-power fluctuations and with respect to spectroscopic fluctuations.

We give two possible implementations for the algorithm to compute the Allan variance spectra which differ in the required computing power and the subjective "smoothness" of the resulting spectra although both are accurate within the achievable uncertainty of the total Allan variance analysis. Although it is usually required to characterise an instrument by a single stability number, we show that the use of an average is not always justified, but has to be checked in each case by visualising the channel-by channel Allan variance. For astronomical applications we propose the "grand average" subtraction scheme providing numbers most relevant for astronomical line observations.

By introducing a new definition of the instrument stability time we can to characterise the instrument also in case of $1 / f$ noise or shallower fluctuation spectra at the cost of not being directly comparable to the traditional Allan minimum time. Using this stability time we compute the optimum observing strategy in symmetric differencing observations for arbitrary drift parameters. If the appropriate loop timing is used, the total uncertainty of the measured spectra per given observing time will be minimised. In case of moderate instrumental dead times relative to the Allan stability time it can guarantee that baseline ripples due to spectroscopic drifts remain hidden in the radiometric noise. If the instrument does not allow the use of the optimum chop cycle the drift noise can exceed the radiometric noise by orders of magnitude. The formalism presented here allows optimization of both total-power and spectroscopic observations, but the considerably lower total-power stability of any instrument demands separate setups for the two different scientific goals.

\subsection{Experience gained from testing HIFI}

The analysis of stability tests of the HIFI instrument showed that gain fluctuations represent the main cause of instrumental instabilities. We found that the temperature and mechanical stability is absolutely critical for a good performance. It turned out that changes in the pump level due to fluctuations in the LO power or standing waves in the optical path between mixer and LO are a major cause of bad Allan stability times as predicted by Siebertz et al. (2002).
This results in first instance in total power instabilities. The total-power Allan times under good conditions can range from a few seconds up to about $20 \mathrm{~s}$ at $2 \mathrm{MHz}$ fluctuation bandwidth. Due to slight impedance mismatches between mixer and amplifiers, any change of the pump level also creates a small change in the spectral response affecting the spectroscopic stability. The standing waves in the LO path also show a characteristic spectral instability imprint. Thus we obtain a reduced spectroscopic stability as second-order byproduct of the gain fluctuations. Under good conditions we measured spectroscopic Allan times exceeding $100 \mathrm{~s}$ at $2 \mathrm{MHz}$ fluctuation bandwidth. When removing first order drifts by difference measurements, the differential Allan variance of the resulting difference spectra measures higherorder instabilities. The available differential schemes result in spectroscopic Allan times of up to a few hundred seconds governing the period of second-order observational loops.

It turns out that spectroscopic binning removes part of the fluctuations seen in the Allan variance spectra, but it enhances the relative contribution of the underlying $1 / f$ noise always present in the gain fluctuations. Consequently, the impact of binning on the Allan time cannot be predicted just from the measured Allan variance spectrum at the native resolution of the instrument. It needs to be measured, but can be fitted by two independent parameters.

The HIFI experience can be used in the same way for ground based telescope when the impact of the atmosphere is included in the Allan variance measurements, i.e. if the stability measurements are performed towards a celestial OFF position. Fluctuations of the atmospheric transmission will again affect mainly the total-power stability, but leave a characteristic spectroscopic pattern in case of narrow absorption features in the observed spectral range. In case of a well-known shape of the transmission function, an appropriate atmospheric model (e.g. Pardo et al. 2004) may be used to correct for this spectroscopic instability.

Acknowledgements. I want to thank Rudolf Schieder, Jacob Kooi, and Oliver Siebertz for direct help in the computations and many useful discussions. Special thanks go to Paul Goldsmith for carefully reviewing the manuscript providing many useful suggestions. This work was supported by the DLR grant $50 \mathrm{OF}$ 0006.

\section{References}

Allan, D. W. 1966, Proc. IEEE, 54, 221

De Graauw, Th., \& Helmich, F. 2000, in The Promise of the Herschel Space Observatory, ed. G. L. Pilbratt, J. Cernicharo, A. M. Heras, T. Prusti, \& A. Harris, ESA SP-460, 45

Kooi, J., Chattopadhyay, G., Thileman, M., Phillips, T., \& Schieder, R. 2000, Int. J. Infrared \& Millim. Waves, 8, 689

Kooi, J. W., Baselmans, J. J., Baryshev, A., et al. 2006, J. Appl. Phys., 100, 64904 Kraus, J. 1980, Radio astronomy (Cygnus Quasar Books), Chap. 7

Kutner, M. L., \& Ulich, B. L. 1981, ApJ, 250, 341

Pardo, J. R., Wiedner, M. C., Serabyn, E., et al. 2004, ApJS, 153, 363

Rohlfs, K., \& Wilson, T. L. 1986, Tools of Radio Astronomy (Berlin: Springer) Schieder, R., \& Kramer, C. 2001, A\&A 373, 746

Schieder, R., Rau, G., \& Vowinkel, B. 1985, in Instrumentation for Submillimetrer Spectroscopy, ed. E. Kollberg (Academic Press), 189

Schieder, R., Tolls, V., \& Winnewisser, G. 1998, Exp. Astron., 1, 101

Schieder R., Siebertz O., Schloeder F., et al. 2000, in UV, Optical, and IR Space Telescopes and Instruments, ed. J. B. Breckinridge, \& P. Jakobsen, SPIE, 4013, 313

Siebertz, O. 1998, Akusto-optisches Spektrometer mit variabler Auflsung, Ph.D. Thesis, Cologne University

Siebertz, O., Honingh, C., Tils, T., et al. 2002, IEEE Transact. Microwave Theory \& Technique, 50,1

Whyborn, N. D. 2003, DM IF-1 Amplifier Gain Stability Summary, SRON Technical Note 06/02/2003 